\title{
Extraskeletal Chondroma of the Index Finger: A Case Report
}

\author{
Masato Saito $^{a} \quad K^{2}$ azumasa Nishimoto ${ }^{a, b} \quad$ Robert Nakayama ${ }^{a}$ \\ Kazutaka Kikuta ${ }^{a}$ Masaya Nakamura $^{a}$ Morio Matsumoto $^{a}$ \\ Hideo Morioka ${ }^{a, c}$ \\ ${ }^{a}$ Department of Orthopaedic Surgery, Keio University School of Medicine, Tokyo, Japan; \\ ${ }^{b}$ Department of Orthopaedic Surgery, Kawasaki Municipal Ida Hospital, Kawasaki, Japan; \\ ${ }^{\mathrm{C}}$ Department of Orthopaedic Surgery, National Hospital Organization Tokyo Medical \\ Center, Tokyo, Japan
}

\section{Keywords}

Extraskeletal chondroma $\cdot$ Cartilaginous tumor $\cdot$ Soft tissue $\cdot$ Finger

\begin{abstract}
Extraskeletal chondroma is defined as a rare, benign, cartilaginous tumor arising from soft tissues such as tendons, tendon sheath synovia, and joint capsules with no continuity to the periosteum or bone cortex. In histopathologic findings, the tumor exhibits many lobular structures and some parts similar to hyaline cartilage. Therefore, it is sometimes difficult to differentiate this tumor from low-grade chondrosarcoma because of their similar histopathologic findings. In order to prevent recurrence, it is necessary to remove the tumor as a whole, including the capsule, so as not to leave any remnants of the tumor. In this article, we report our treatment experience with a case of extraskeletal chondroma in the index finger of a 63year-old patient.




\section{Case Reports in Oncology}

Saito et al.: Extraskeletal Chondroma of the Index Finger: A Case Report

\section{Introduction}

Extraskeletal chondroma is a rare, benign, cartilaginous tumor that develops in soft tissues and is not attached to the periosteum or bone cortex [1]. In this article, we report our treatment experience with a case of extraskeletal chondroma occurring in the index finger.

\section{Case Report}

The patient was a 63-year-old male. Around 2006, he became aware of a mass on the palmar side of the middle phalanx of the left index finger. Thereafter, although the mass gradually became larger, it was left untreated. In May 2010, he underwent magnetic resonance imaging (MRI) at another hospital and was diagnosed with a soft-tissue tumor in the left index finger. In June 2010, he was referred to our department. An elastic, hard, immobile mass of about $4 \mathrm{~cm}$ in diameter with slight redness and not accompanied by tenderness or a sensation of heat was found in the left index finger (Fig. 1). In addition, markedly limited flexion ( 0 degree) at the distal interphalangeal joint of the left index finger was observed. There were no abnormal findings in hematology and urinalysis.

Plain radiography revealed a tumor shadow on the palmar side of the middle phalanx of the left index finger, with faint internal calcification. Lateral images showed no obvious bone destruction, with a slight indentation on the palmar surface of the middle phalanx (Fig. 2). MRI revealed a $40 \times 38-\mathrm{mm}$ mass shadow along the flexor tendon. The mass was found to be a well-demarcated tumor, showing uneven isointensity and low-signal intensity on T1weighted images (Fig. 3a) and uneven high- and low-signal intensity on T2-weighted images (Fig. 3b, c). On both T1- and T2-weighted images, low-signal regions mean calcification in the tumor. With the findings of the faint calcification on plain radiography and the high- and low-signal intensity on T2-weighted images of MRI, the imaging-based diagnosis was cartilaginous tumor. In addition, since no obvious malignant findings such as bone destruction or invasion were obtained by imaging, the tumor was considered benign. On November 17, 2010, marginal tumor resection was performed, also aiming to make a histological diagnosis. The tumor was found to have arisen from the flexor tendon sheath (A2, A3 pulley). Therefore, the tumor was removed along with the tendon sheath.

The macroscopic findings of the resected specimens revealed a white, well-demarcated tumor of about $4 \mathrm{~cm}$ in size. Histopathological examination revealed the presence of mature hyaline cartilage across the tumor mass, with a relatively low cell density and small mononuclear chondrocytes with no apparent atypia in the cartilage space (Fig. 4). Necrotic and binuclear cells were scarce, and there was no involvement of the periosteum. Therefore, the final diagnosis was determined as extraskeletal chondroma. As of 6 years and 5 months after the operation, although the mild flexion contracture of the distal interphalangeal joint of the left index finger remains, it does not interfere with everyday life, and no local recurrence has been observed.

\section{Discussion}

Extraskeletal chondroma was described in detail by Enzinger in 1983 [2]. It is defined as a benign cartilaginous tumor arising from soft tissues such as tendons, tendon sheath synovia, and joint capsules with no continuity to the periosteum or bone cortex. Cases of ex- 
traskeletal chondroma are estimated to account for about 1.5\% of all benign soft-tissue tumors [3]. Thus, it is a rare tumor. Extraskeletal chondroma typically affects adults between 30 and 60 years of age, and there is no obvious gender difference [1, 2]. The most common sites of occurrence are the fingers and toes [3]. According to a study of 104 cases reported by Chung and Enzinger [4], extraskeletal chondroma occurred in fingers in about $80 \%$ of the cases. Therefore, various symptoms related to the hands and fingers by the tumor were reported $[5,6]$. Extraskeletal chondroma also occurs in the trunk, and rarely in the dura mater, pharynx, mouth, and skin. The tumor is well-demarcated and oval in shape, rarely with a diameter exceeding $3 \mathrm{~cm}$ [7].

The first choice of treatment is marginal tumor resection. However, the local recurrence rate with this procedure is relatively high. According to a report by Dahlin and Salvador [1], the recurrence rate was about $17 \%$. In order to prevent recurrence, it is necessary to remove the tumor as a whole, including the capsule, so as not to leave any remnants of it.

Histopathologically, the tumor exhibits many lobular structures and some parts similar to hyaline cartilage containing mucous components. In the matrix of the tumor, spindleshaped or circular eosinophilic cells exist in an irregular and sparse fashion. Tumor cells become round in shape and swollen, forming a structure similar to hyaline cartilage. In some cases, incomplete lacuna-like cavities are formed in the surrounding matrix. Even benign chondroma has nuclear atypia and multinuclear cells in some cases. Therefore, histopathological differential diagnosis from low-grade chondrosarcoma is sometimes required.

Clinical findings and histopathological differential diagnosis include calcifying aponeurotic fibroma, giant cell tumor of the tendon sheath, periosteal chondroma, and low-grade chondrosarcoma. It is sometimes difficult to differentiate extraskeletal chondroma from periosteal chondroma. However, in the present case, the tumor was identified during the operation to have arisen from the tendon sheath, and this led us to make the diagnosis of extraskeletal chondroma. This tumor is also sometimes difficult to be histologically differentiated from low-grade chondrosarcoma because of their similar histopathologic findings. In the present case, the tumor was diagnosed as a benign, cartilaginous tumor based on the findings of no increase in cell density, scarce necrotic and binuclear cells, and small mononuclear chondrocytes with no apparent atypia in the cartilage space.

\section{Statement of Ethics}

The authors have no ethical conflicts to disclose.

\section{Disclosure Statement}

The authors have no conflicts of interest to declare.

\section{References}

1 Dahlin DC, Salvador AH: Cartilaginous tumors of the soft tissues of the hands and feet. Mayo Clin Proc 1974;49:721-726.

2 Enzinger FM: Cartilaginous tumors and tumor-like lesions of soft tissue; in Enzinger FM, Weiss SW (eds): Soft Tissue Tumors. St. Louis, Mosby Co, 1983, pp 698-719.

3 Kransdorf M, Meis J: Extraskeletal osseous and cartilaginous tumors of the extremities. Radiographics 1993;13:853-884. 


\section{Case Reports in Oncology}

4 Chung EB, Enzinger FM: Chondroma of soft parts. Cancer 1978;41:1414-1424.

-5 Schwaiger K, Ensat F, Neureiter D, Wechselberger G, Hladik M: Trigger finger caused by extraskeletal chondroma. J Hand Surg Am 2017;42:e51-e55.

-6 Ishii T, Ikeda M, Oka Y: Subungual extraskeletal chondroma with finger nail deformity: case report. J Hand Surg Am 2010;35:296-299.

7 Cartilaginous and osseous soft tissue tumors; in Goldblum JR, Folpe AL, Weiss SW (eds): Soft Tissue Tumors, ed 6. Philadelphia, Elsevier Saunders, 2014, pp 917-946.

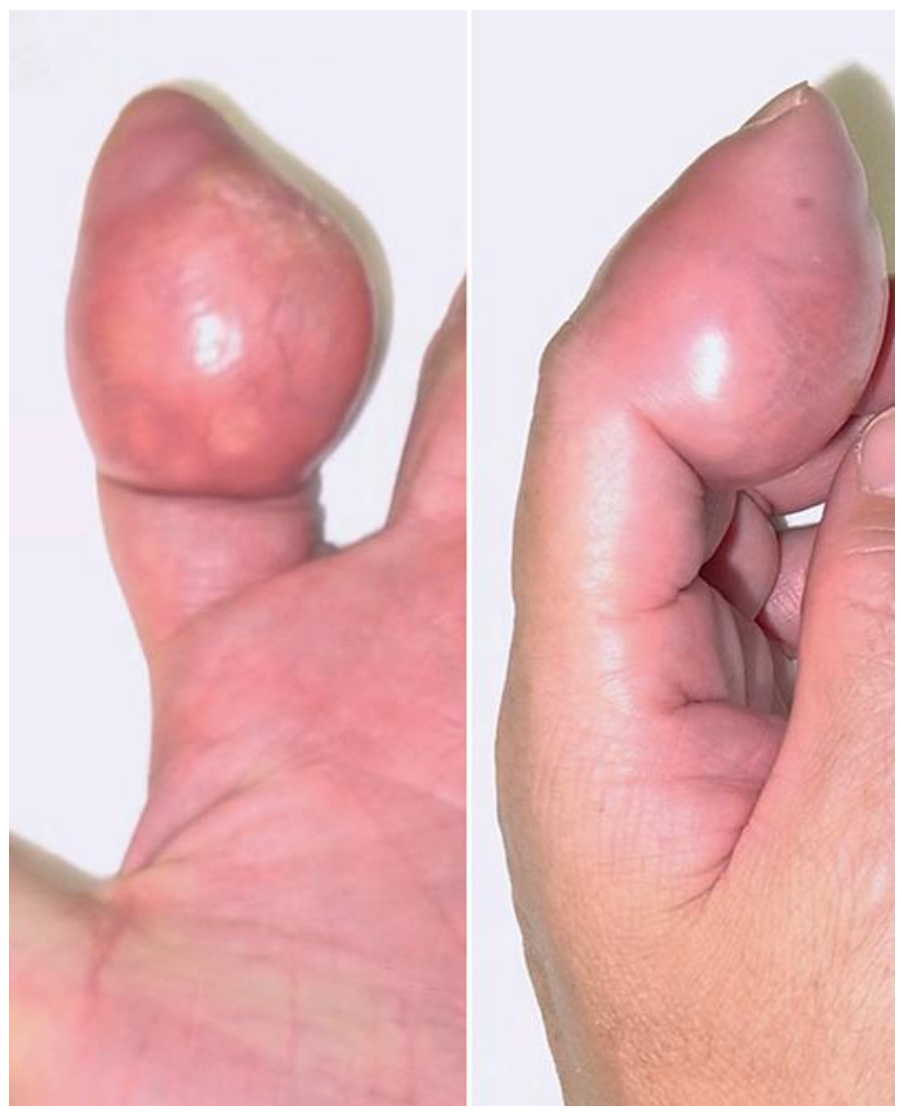

Fig. 1. A mass of about $4 \mathrm{~cm}$ in diameter with slight redness in the left index finger. 


\section{Case Reports in Oncology}

\begin{tabular}{l|l}
\hline Case Rep Oncol 2017;10:479-484 \\
\hline DOI: 10.1159/000477237 & $\begin{array}{l}\text { @ } 2017 \text { The Author(s). Published by S. Karger AG, Basel } \\
\text { www.karger.com/cro }\end{array}$ \\
\hline
\end{tabular}

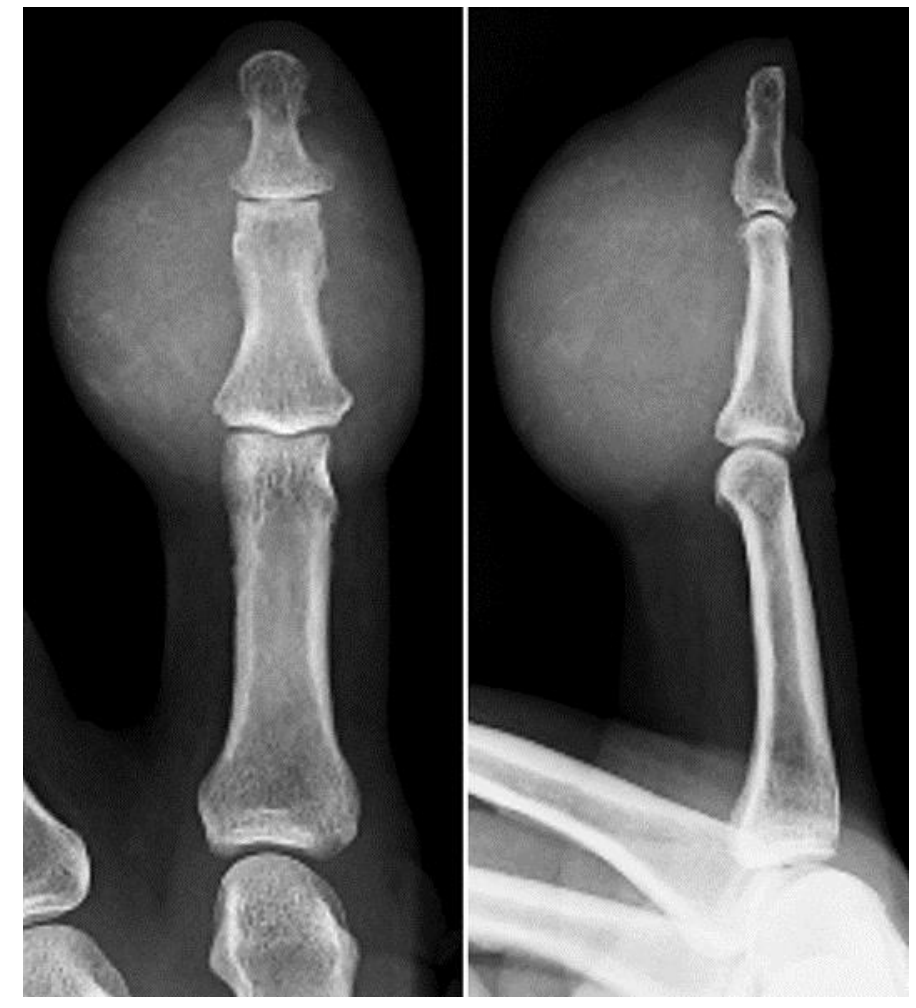

Fig. 2. Plain radiography showing a tumor shadow on the palmar side of the middle phalanx of the left index finger, with faint internal calcification.
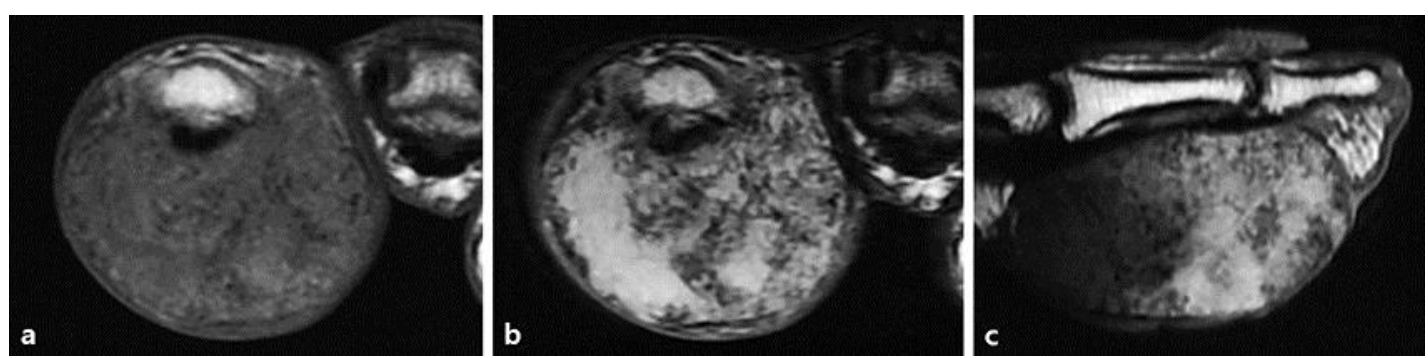

Fig. 3. Magnetic resonance imaging revealing a $40 \times 38-\mathrm{mm}$ mass shadow along the flexor tendon. The mass is found to be a well-demarcated tumor, showing uneven isointensity and low-signal intensity on T1weighted images (a) and uneven high- and low-signal intensity on T2-weighted images (b, c). On both T1and T2-weighted images, low-signal regions mean calcification in the tumor. 


\section{Case Reports in Oncology}

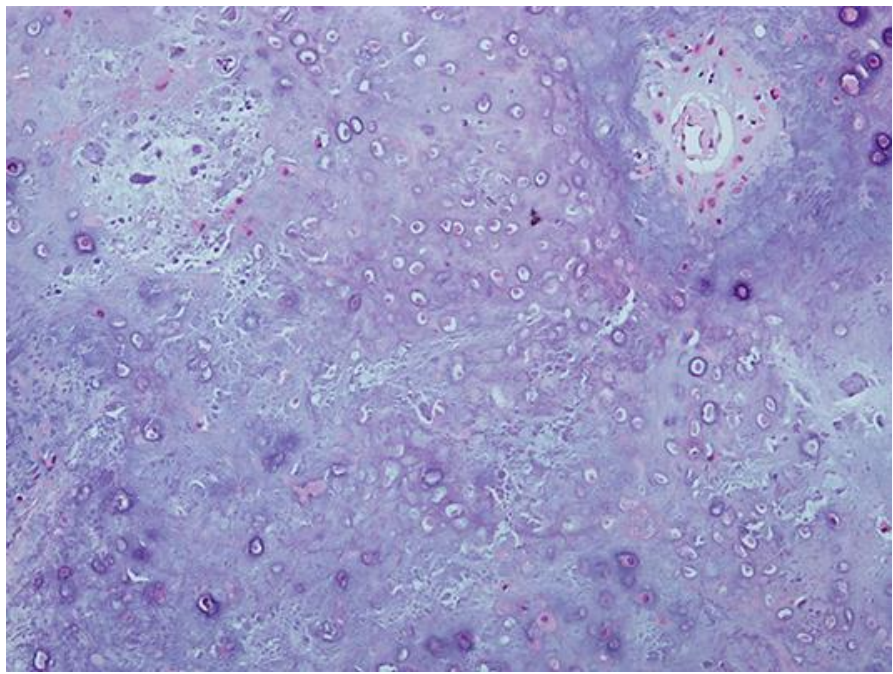

Fig. 4. Histopathological findings showing the presence of mature hyaline cartilage across the tumor mass, with a relatively low cell density and small mononuclear chondrocytes with no apparent atypia. 